\title{
Optimal Performance Analysis of Feynman's Microscopic Heat Engine
}

\author{
Yuling Xiao, a , Jizhou $\mathrm{He}^{2, b}$ and Haitao Cheng ${ }^{3, c}$ \\ ${ }^{1}$ Department of Physics, Nanchang University, Nanchang, P. R. China \\ ${ }^{2}$ Department of Physics, Nanchang University, Nanchang, P. R. China \\ ${ }^{3}$ Department of Physics, Nanchang University, Nanchang, P. R. China \\ axyl_76@sohu.com, ${ }^{\text {b }}$ hjzhou@ncu.edu.cn, ${ }^{c}$ chenghaitao2007@126.com
}

Keywords: Feynman's microscope heat engine, Stochastic motion, Heat leak, Finite time thermodynamics.

Abstract. The model of a Feynman's microscope heat engine in a one-dimensional lattice is established in this paper. Based on stochastic master equation, the expressions of the current, efficiency and power output of the heat engine are derived analytically. The performance characteristic curves of the power output versus the efficiency are the loop-shaped ones. It shows that the heat engine is irreversible and its efficiency is far less than the Carnot efficiency. Moreover, the influence of the height of barrier and the temperature ratio on the optimal performance of heat engine is analyzed. The heat engine can work at the optimal operation regime by reasonablly choosing the external parameters.

\section{Introduction}

As the size of a system approaches that of molecules, thermal fluctuations begin to play a significant role. It would be rather difficult to operate a nanoscale machine against strong thermal fluctuations. Instead, the nanomachine must be able to work harmoniously or collaboratively with the fluctuations. In order to design such machines, we need to understand thermodynamics of small systems, taking into account large thermal fluctuations. Recently, the study of microscopic heat engines rectifying thermal fluctuations has attracted considerable attention [1-8], including Brownian heat engines, stochastic heat engines, diode engines, and so on. One typical model of the microscopic engines rectifying thermal fluctuations is Feynman's ratchet and pawl engine[9-11]. Feynman et al. [9] attempted to investigate the thermodynamics of the Feynman-Smoluchowski (FS) motor and concluded that it can reach Carnot efficiency. But later it was shown that the heat transfer between two heat reservoirs never ceases even when the motor moves quasistatically due to the effect of thermal fluctuations. Thus, it is not possible to reach the Carnot efficiency [10]. Corresponding to the discrete and classical model of Feynman's ratchet and pawl engine, a continuous and microscopic model of Feynman's heat engine was proposed by Jarzynski $\mathrm{C}$ et al [12]. It showed that this microscopic model can act both as a heat engine or a refrigerator and its efficiency or coefficient of performance is far less than Carnot value. Following their work, we further study the optimal performance of the heat engine in this paper. Based on stochastic master equation, the expressions of the current, efficiency and power output of the heat engine are derived analytically. The performance characteristic curves of the power output versus the efficiency are plotted. Then, the influence of the height of barrier and the temperature ratio on the optimal performance parameters is analyzed.

\section{Model and Theory}

Consider a particle that jumps between neighboring sites along a one-dimensional regular lattice, where $d$ is the lattice spacing. We assume that the potential energy function $U_{i}^{(m)}$ has two possible modes, $m=1,2$, and that it changes stochastically between these two. The expression of the potential energy function is given by 


$$
U_{i}^{(m)}=\left\{\begin{array}{l}
U_{i}^{(1)}=0, \quad m=1 \\
U_{i}^{(2)}=E[i(\bmod ) 3-1], \quad m=2
\end{array}\right.
$$

where $E$ is a positive constant with units of energy. When an external load is added to the particle, the potential energy function $U_{i}^{(m)}$ is given by $U_{i}^{(m)} \rightarrow U_{i}^{(m)}+i f d$ where $f$ is a real constant. The second mode is a discrete version of an asymmetric sawtooth potential, as shown in Fig. 1. We assume that the particle is coupled to a hot reservoir at temperature $T_{h}$, and that its jumps are thermal in nature. Thus, the probability of making a jump to site $i+1$, starting from site $i$, is related to the rate of the inverse process by the usual detailed balance relation: $\exp \left[-\left\{\left(U_{i+1}^{(m)}-U_{i}^{(m)}\right)+f d\right\} / k_{B} T_{h}\right]$, where $f$ is the external load. We assume that the stochastic process governing the changes between modes is also a thermal process, driven by a cold reservoir at temperature $T_{c}$. Thus, the rate of a change to mode 2 , starting from mode 1 , is related to the rate of the reverse mode change by the detailed balance factor $\exp \left[-\left(U_{i}^{(2)}-U_{i}^{(1)}\right) / k_{B} T_{c}\right]$.

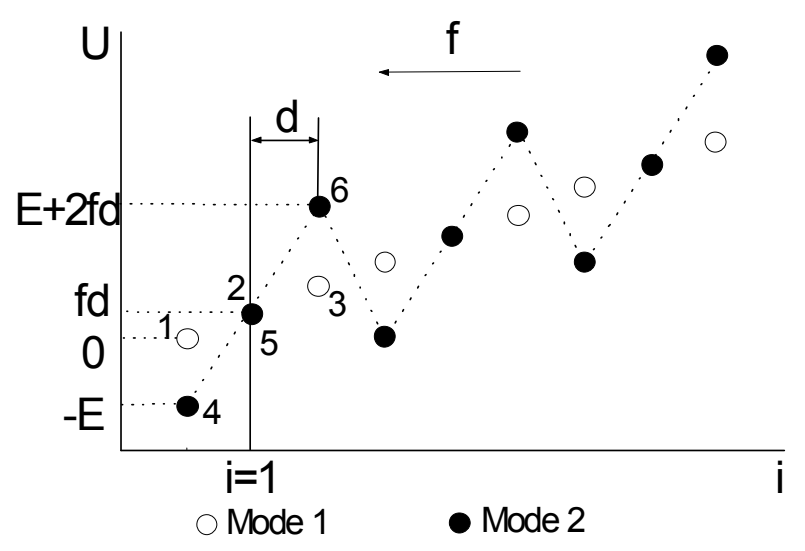

Fig.1 Schematic diagram of the potential energy $U_{i}^{(m)}$ for both modes $m=1,2$.

It is seen from Fig. 1 that the modle has six possible states during a period. The dynamics of the particle is described by stochastic master equation[12]:

$\frac{d \vec{p}}{d t}=\Gamma \mathfrak{R} \vec{p}, \quad \vec{p}=\left(p_{1}, p_{2}, \ldots, p_{6}\right)^{T}$,

$\Re=\left[\begin{array}{cccccc}-\frac{3}{2}-\frac{\sigma}{2} & \frac{1}{2} & \frac{\sigma}{2} & \mu & 0 & 0 \\ \frac{\sigma}{2} & -\frac{3}{2}-\frac{\sigma}{2} & \frac{1}{2} & 0 & 1 & 0 \\ \frac{1}{2} & \frac{\sigma}{2} & -\frac{1}{2}-\mu-\frac{\sigma}{2} & 0 & 0 & 1 \\ 1 & 0 & 0 & -\mu-\frac{v^{2}}{2 \sigma}-\frac{v \sigma}{2} & \frac{1}{2} & \frac{1}{2} \\ 0 & 1 & 0 & \frac{v \sigma}{2} & -\frac{3}{2}-\frac{v \sigma}{2} & \frac{1}{2} \\ 0 & 0 & \mu & \frac{v^{2}}{2 \sigma} & \frac{v \sigma}{2} & -2\end{array}\right]$ 
where $\mu=\exp \left(-E / k_{B} T_{c}\right), v=\exp \left(-E / k_{B} T_{h}\right), \sigma=\exp \left(-f d / k_{B} T_{h}\right), \Gamma$ is the rate that the particle attempts a jump per unit time and $p_{n}(n=1, \ldots, 6)$ is the probability of finding the particle at state $n$ at time $t . \mathfrak{R}_{n n^{\prime}}\left(n, n^{\prime}=1, \ldots, 6\right)$, given by the Metropolis rule, is the transition probability that the particle jumps from state $n^{\prime}$ to $n$. The steady-state probability distribution $\bar{p}_{n}(n=1, \ldots, 6)$ of Eq. 2 can be obtained by solving $d \vec{p} / d t=0$ (i.e., $\mathfrak{R} \vec{p}=0$ ). The explicit expression for the steady-state current is given by

$$
J=\Gamma\left(\bar{p}_{1} \Re_{21}+\bar{p}_{4} \Re_{54}-\bar{p}_{2} \mathfrak{R}_{12}-\bar{p}_{5} \Re_{45}\right)
$$

The drift velocity $v$ is given by $v=3 d J$. Whenever the particle jumps from state 4 to 5 , from state 4 to 6 and from state 5 to 6 , it takes heat from the hot reservoir. When the particle jumps from state 5 to 4 , from state 6 to 4 and from state 6 to 5 , it gives heat to the hot reservoir. Thus, the heat flow transferred from the hot reservoir is

$\dot{Q}_{h}=E \Gamma\left(\bar{p}_{4} \Re_{54}+2 \bar{p}_{4} \Re_{64}+\bar{p}_{5} \Re_{65}-\bar{p}_{5} \Re_{45}-\bar{p}_{6} \mathfrak{R}_{56}-2 \bar{p}_{6} \Re_{46}\right)+3 f d \Gamma J$

The heat flow released to the cold reservoir is

$$
\dot{Q}_{c}=E \Gamma\left(\bar{p}_{1} \Re_{41}+\bar{p}_{6} \Re_{36}-\bar{p}_{4} \Re_{14}-\bar{p}_{3} \Re_{63}\right)
$$

In the steady-state there is a equivalent relation

$$
\bar{p}_{4} \Re_{54}+2 \bar{p}_{4} \Re_{64}+\bar{p}_{5} \Re_{65}-\bar{p}_{5} \Re_{45}-\bar{p}_{6} \Re_{56}-2 \bar{p}_{6} \Re_{46}=\bar{p}_{1} \Re_{41}+\bar{p}_{6} \Re_{36}-\bar{p}_{4} \Re_{14}-\bar{p}_{3} \Re_{63}
$$

The power output and efficiency are

$$
P=\dot{Q}_{h}-\dot{Q}_{c}=3 f d \Gamma J
$$

And

$$
\eta=\frac{P}{\dot{Q}_{h}}=\frac{3 \lambda \Gamma J}{a \Gamma\left(\bar{p}_{4} \Re_{54}+2 \bar{p}_{4} \Re_{64}+\bar{p}_{5} \Re_{65}-\bar{p}_{5} \Re_{45}-\bar{p}_{6} \mathfrak{R}_{56}-2 \bar{p}_{6} \mathfrak{R}_{46}\right)+3 \lambda \Gamma J}
$$

respectively. After introducing three dimensionless parameters, i.e. the barrier height $a=E / k_{B} T_{c}$, the temperature ratio $\tau=T_{h} / T_{c}$ and the external load $\lambda=f d / k_{B} T_{c}$, Eq. 4, 5 and 7 may be rewritten as

$$
\begin{aligned}
\dot{Q}_{h}^{*} & =\frac{\dot{Q}_{h}}{T_{c}}=a \Gamma\left(\bar{p}_{4} \Re_{54}+2 \bar{p}_{4} \Re_{64}+\bar{p}_{5} \Re_{65}-\bar{p}_{5} \Re_{45}-\bar{p}_{6} \Re_{56}-2 \bar{p}_{6} \Re_{46}\right)+3 \lambda \Gamma J \\
\dot{Q}_{c}^{*}= & \frac{\dot{Q}_{c}}{T_{c}}=a \Gamma\left(\bar{p}_{1} \Re_{41}+\bar{p}_{6} \Re_{36}-\bar{p}_{4} \Re_{14}-\bar{p}_{3} \Re_{63}\right) \\
P^{*} & =P / k_{B} T_{c}=3 \lambda \Gamma J
\end{aligned}
$$


For the convenience of calculation, we make $\Gamma=1, k_{B}=1$ and $d=1$.

\section{General Performance Characteristics}

The curves of heat flow $\dot{Q}_{h}{ }^{*}$ and the drift velocity $v$ versus the external load $\lambda$ for given $a$ and $\tau$ are plotted, as shown in Fig. 2. It is seen that the heat flow and drift velocity both decrease monotonically as $\lambda$ increases. When $0<\lambda<\lambda_{0}$, the drift velocity is positive and the particle drifts rightward. Thus, the model can work as a heat engine. When $\lambda>\lambda_{0}$, the particle drifts leftward and the heat engine loses its role. When $\lambda=\lambda_{0}$, the drift velocity is zero, but the heat flow has a positive value $\dot{Q}_{k}{ }^{*}$ and $\dot{Q}_{h}{ }^{*}=\dot{Q}_{c}{ }^{*}$. This means that it exists a nonzero heat flow $\dot{Q}_{k}{ }^{*}$, which is similar to the heat leak transferred from hot reservoir to cold reservoir in the heat engine. [13-14].

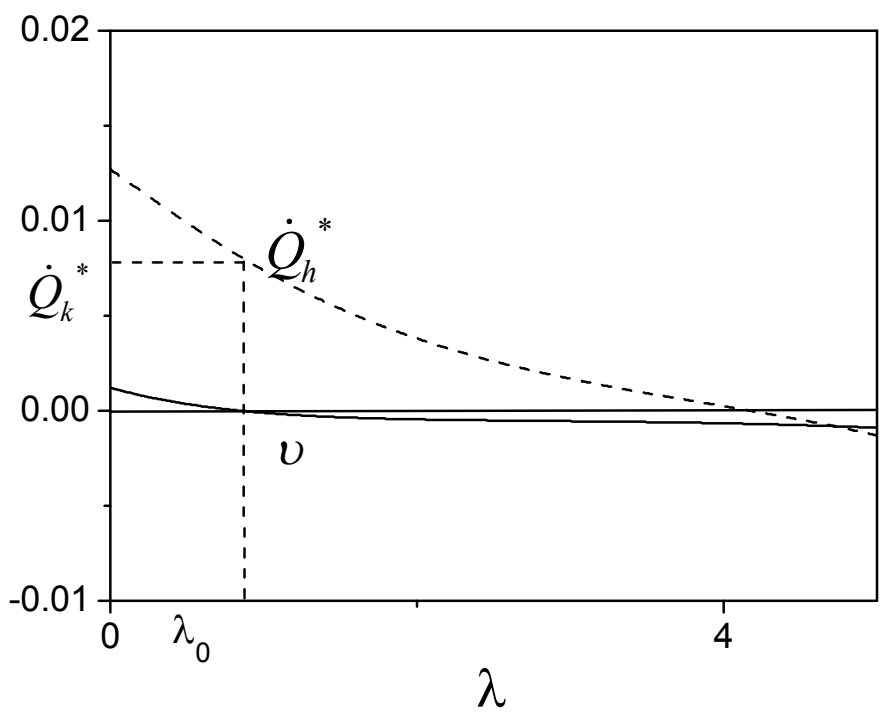

Fig. 2 the heat flow $\dot{Q}_{h}{ }^{*}$ and drift velocity $v$ versus the external load $\lambda$ at given $a=10$ and $\tau=2$.

Using Eq. 8 and 11, one can generate the curves of the power output and the efficiency versus the external load $\lambda$ at different $a$ for given $\tau=2$, as shown in Fig. 3 and 4. It can be seen that the power output and the efficiency both first increase and then decrease as the external load $\lambda$ increases. There exist both a maximum power output $P^{*}{ }_{\text {max }}$ at the special value $\lambda_{P^{*}}$ and a maximum efficiency $\eta_{\max }$ at the special value $\lambda_{\eta}$. Both maximum power output $P^{*}{ }_{\max }$ and maximum efficiency $\eta_{\max }$ decrease as the barrier height $a$ increases. The curves of the power output versus the efficiency are plotted at different $a$, as shown in Fig. 5. The performance characteristic curve is a loop-shaped one. The optimal operating regions of the heat engine should be located in those of the $P \sim \eta$ curve with a negative slope. It is clearly shown that when the power is in this region, there are two different efficiencies for a given power output, where one is smaller than $\eta_{P}$ and the other is larger than $\eta_{P}$. Obviously, one always wants to obtain the efficiency as large as possible for a given power. Thus, the optimal efficiency should be

$$
\eta_{P} \leq \eta \leq \eta_{\max }
$$


$\eta_{P}$ and $\eta_{\max }$ are two important performance parameters which determine the lower and upper bounds of the optimal efficiency. The optimal power output should be

$$
P^{*}{ }_{\eta} \leq P^{*} \leq P^{*}{ }_{\max }
$$

$P^{*}{ }_{\eta}$ and $P^{*}{ }_{\text {max }}$ are two important performance parameters which determine the lower and upper bounds of the optimal efficiency.

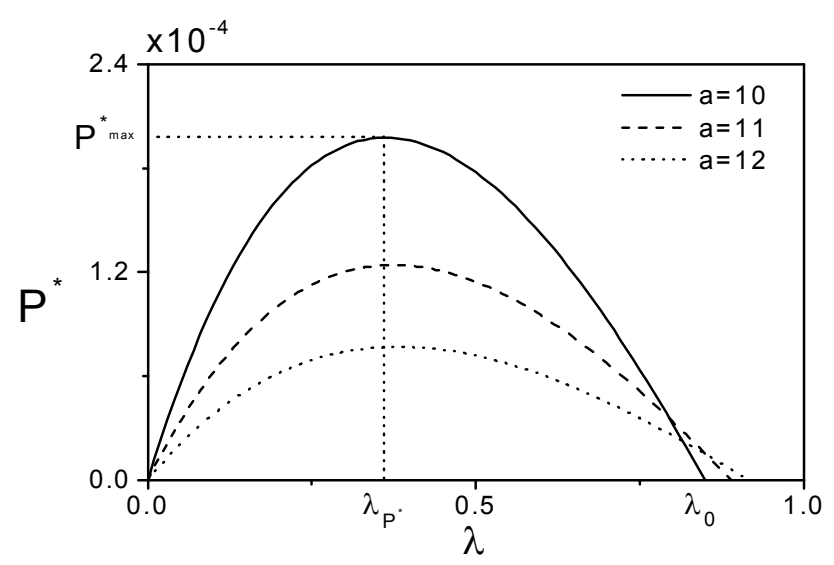

Fig.3 The dimensionless power output $P^{*}$ versus the external load $\lambda$ for given $\tau=2$.

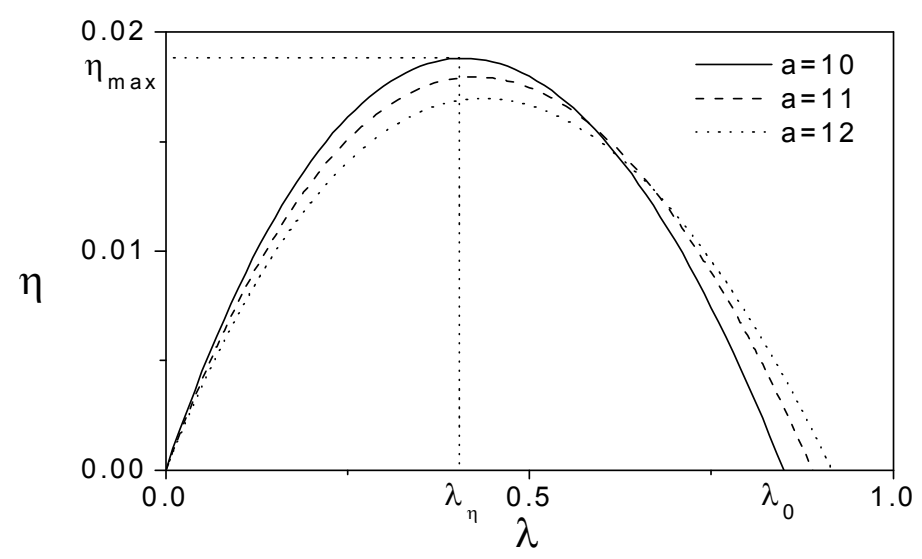

Fig.4 The efficiency $\eta$ versus the external load $\lambda$ for given $\tau=2$.

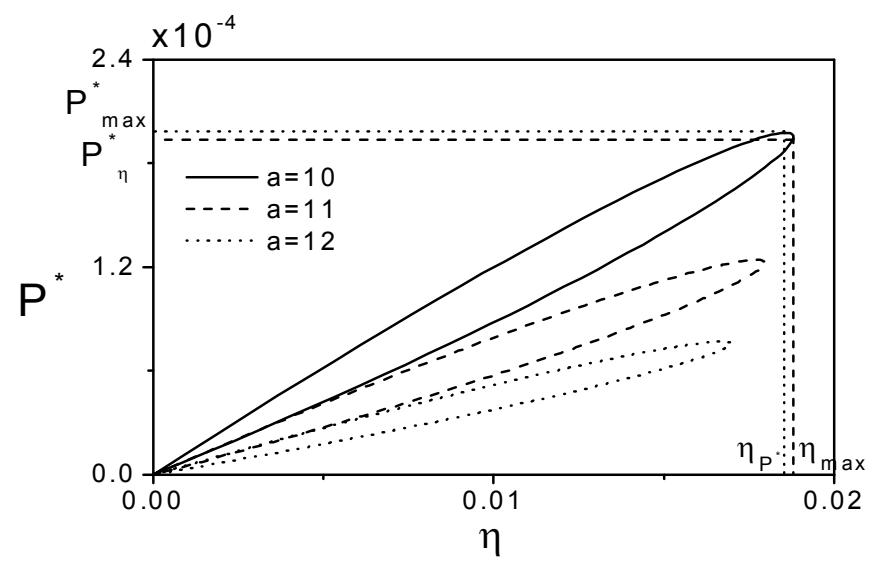

Fig.5 The dimensionless power output $P^{*}$ versus efficiency $\eta$ for given $\tau=2$. 


\section{Influence of the Barrier Height and the Temperature Ratio of Optimal Performance Parameters}

Using Eq. 8 and 11 as well as the extremal conditions $\partial P^{*} / \partial \lambda=0$ and $\partial \eta / \partial \lambda=0$, one can generate the curves of the maximum power output $P^{*}{ }_{\max }$ and the maximum efficiency $\eta_{\max }$ versus the parameter $\tau$ at different $a$, as shown in Fig. 6 . It can be seen that both the maximum power output and the maximum efficiency first increase and then decrease as $\tau$ increases. There exist an optimized value $P_{\text {opt }}^{*}$ of maximum power output at $\tau=\tau_{P^{*} \text { opt }}$ and an optimized value $\eta_{\text {opt }}$ of maximum efficiency at $\tau=\tau_{\eta^{*} \text { opt }}$, respectively. When $\tau=1$, the engine won't work, $P^{*}{ }_{\max }$ and $\eta_{\max }$ approach to zero. Through the choice of the parameters $a$ and $\tau$, we can make our heat engine work in the optimal performance regimes. Similarly, one can generate the curves of the power output $P^{*}{ }_{\eta}$ at maximim efficiency and the efficiency $\eta_{P}$ at maximum power output versus the parameter $\tau$ at different $a$, as shown in Fig. 7.
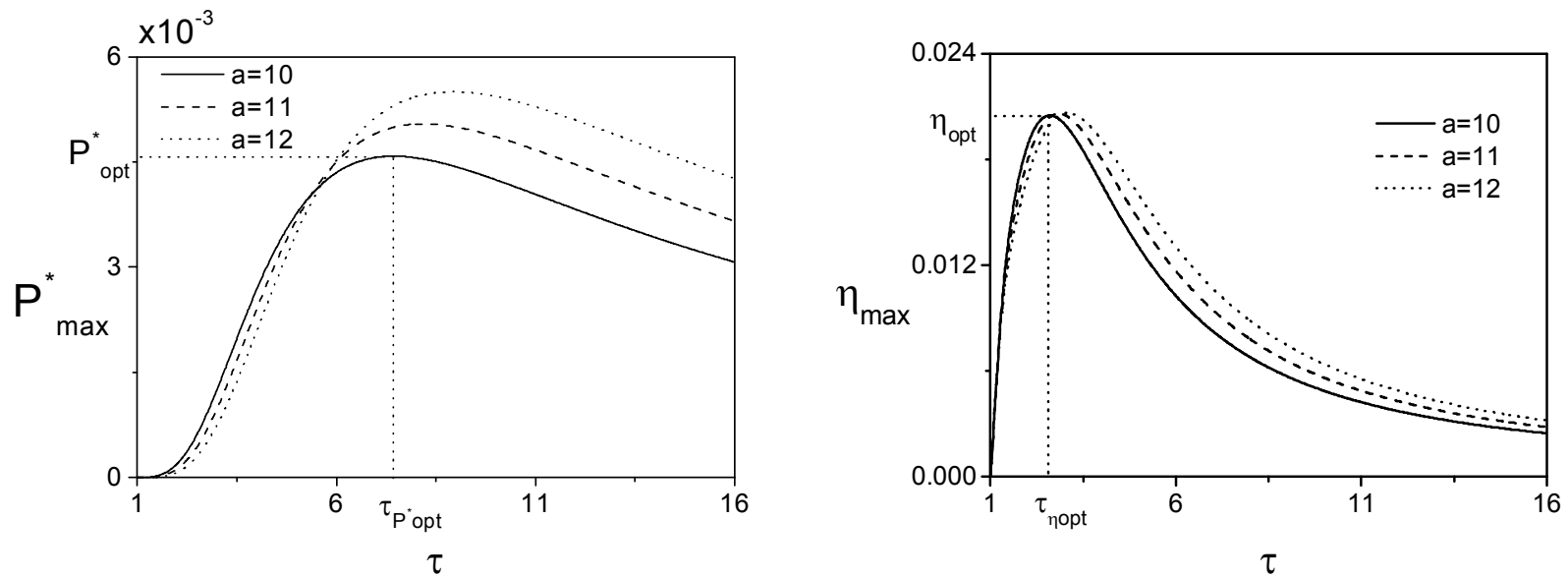

Fig.6 The maximum power output $P^{*}{ }_{\max }$ and the maximum efficiency $\eta_{\max }$ versus the temperature ratio $\tau$.
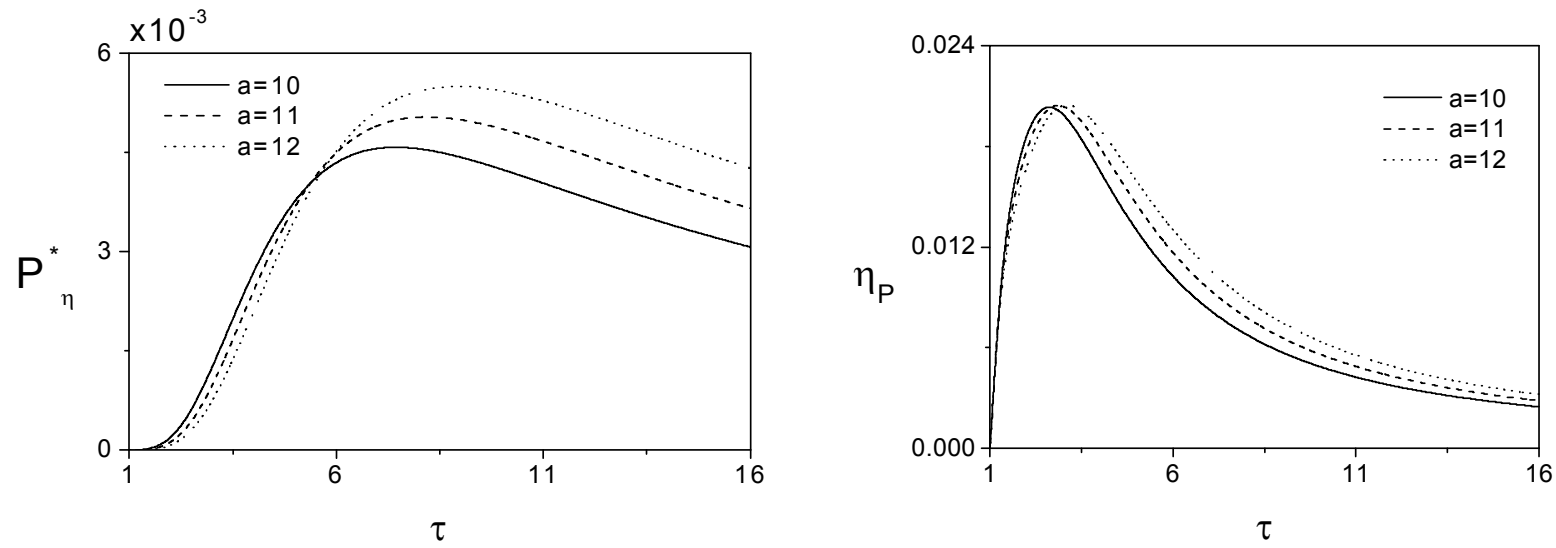

Fig.7 The power output $P^{*}{ }_{\eta}$ at the maximum efficiency and the efficiency $\eta_{P}$ at the maximum power output versus the temperature ratio $\tau$. 


\section{Conclusions}

A simple and discrete model of Feynman's microscope heat engine in one-dimensional lattice has been established. The influence of some external parameters such as barrier height and temperature ratio on the optimal performance of the heat engine has been analyzed. The major results we have obtained: (i) there is an irreversible heat leak between two reservoirs, which is a monotonically increasing function of temperature ratio; (ii) The efficiency is far lower than the Carnot efficiency, and the power-efficiency curve is a loop shape, similar to that in real heat engine; (iii) Feynman's heat engine can work at the optimal operation regime by reasonably choosing the external parameters.

\section{Acknowledgements}

This work was supported by the National Natural Science Foundation of China (Grant No. 11365015).

\section{References}

[1] P. Reimann and P. Häanggim: Appl. Phys. A: Mater. Sci. Proce Vol. 75 (2002), p. 169

[2] C. Van den Broeck and R. Kawai: Phys. Rev. Lett. Vol. 96 (2006), p. 210601

[3] M. Asfaw and M. Bekele: Phys. Rev. E Vol.72 (2005), p. 056109

[4] R. Benjamin and R. Kawai: Phys. Rev. E Vol. 77 (2008), p. 051132

[5] B. H. Lin and J. C. Chen: J. Phys. A: Math. Theor Vol. 42 (2009), p. 075006

[6] Z. M. Ding, L. G. Chen and F. R. Sun: Braz. J. Phys. Vol. 40 (2010), p. 141

[7] Y. P. Zhang, J. Z. He, X. He and Y. L. Xiao: Commun. Theor. Phys. Vol. 54 (2010), p. 857

[8] N. Salas Sanchez and A. Calvo Hernandez: J. Phys. D: Appl. Phys. Vol. 35 (2002), p. 1442

[9] R. P. Feynman, R. B. Leighton and M. Sands: The Feynman Lectures on Physics (Addison-Wesley Publishing Company, New Jersey 1966)

[10] S. Velasco, J. M. M. Roco, A. Medina and A. Calvo Hernandez: J. Phys. D: Appl. Phys. Vol. 34 (2001), p. 1000

[11] Y. Zhang, B. H. Lin and J. C. Chen: Eur. Phys. J. B. Vol. 53 (2006), p. 481

[12] C. Jarzynski and O. Mazonka: Phys. Rev. E Vol. 59 (1999), p. 6448

[13] J. M. Gordon and M. Huleihil: J. Appl. Phys. Vol. 72 (1992), p. 829

[14] J. C. Chen and Z. J. Yan: J. Phys. D: Appl. Phys. Vol. 29 (1996), p. 987 